\title{
Effect of a Magnetic Field in Simulating the Very Near-Field Plume of an Anode Layer Hall Thruster
}

\author{
Yongjun Choi ${ }^{1}$, Michael Keidar ${ }^{2}$ and Iain D. Boyd ${ }^{3}$ \\ Uinversity of Michigan, Ann Arbor, MI 48109
}

\begin{abstract}
Two dimensional axi-symmetric simulations of xenon plasma plume flow fields from a D55 anode layer Hall thruster are performed with a hybrid particle-fluid method. The magnetic field surrounding the Hall thruster exit is included in the calculation. In this simulation, the Boltzmann model and a detailed fluid model are used to compute the electron properties, the direct simulation Monte Carlo method models the collisions of heavy particles, and the Particle-In-Cell method models the transport of ions in an electric field. The accuracy of the simulation is assessed through comparison with various measured data. It is found that a magnetic field significantly affects the profile of the plasma in the Detailed model. For instance, in the case of zero magnetic field, the plasma has a potential about $110 \mathrm{~V}$ at $10 \mathrm{~mm}$ from the thruster exit, while in the case of a magnetic field included, the plasma potential is about $80 \mathrm{~V}$. Results predicted by the Detailed model with the magnetic field are found to be in better agreement with experimental data.
\end{abstract}

\section{Nomenclature}

$\mathrm{B}=$ magnetic field vector

$c_{e} \quad=$ mean electron thermal velocity

$C_{i} \quad=$ ionization coefficient

$\mathrm{E} \quad=$ electric field Vector

$e \quad=$ unit charge

$g \quad=$ relative velocity

$k=$ Boltzmann constant

$m_{e} \quad=$ electron mass

$m_{i} \quad=$ ion mass

$n_{a} \quad=$ atom number density

$n_{e} \quad=$ electron number density

$n_{\max } \quad=$ local maximum of number density

$p_{e} \quad=$ electron pressure

$T_{e} \quad=$ electron temperature
$T_{H}=$ heavy particle temperature

$\mathrm{V}_{\text {drift }}=$ electron drift velocity vector

$\mathrm{V}_{\mathrm{e}}=$ electron velocity vector

$\mathrm{V}_{\mathrm{i}}=$ ion velocity vector

$\sigma=$ plasma conductivity

$\sigma_{i}=$ reference cross section for xenon

$\varepsilon_{i}=$ ionization energy

$\kappa_{e}=$ electron thermal conductivity

$v_{e}=$ electron collision frequency

$v_{e i}=$ ion-electron collision frequency

$v_{e n}=$ neutral electron collision frequency

$\phi=$ plasma potential

$\Psi=$ electron stream function

\section{Introduction}

$\mathrm{H}$ thrust levels, e.g. station-keeping, orbit raising, and orbit transfers. In a Hall thruster, ions are accelerated by electric fields and used to generate propulsive thrust. The energy required to accelerate ions is obtained from on-board batteries or solar cells. Hall thrusters are able to perform better than chemical propulsion systems because Hall thrusters can obtain electricity input directly in space through solar cells, do not need to carry any oxidizer, and so

\footnotetext{
${ }^{1}$ Graduate student, Department of Aerospace Engineering, AIAA Student Member.

${ }^{2}$ Assistant Research Scientist, Department of Aerospace Engineering, AIAA Senior Member.

${ }^{3}$ Professor, Department of Aerospace Engineering, AIAA Associate Fellow.
} 
allow a larger payload. Furthermore, Hall thrusters can realize much higher propellant exhaust velocities than chemical propulsion systems, thereby achieving higher impulse from a given propellant mass and making the use of Hall thrusters for interplanetary missions feasible.

In the past years, two types of Hall thrusters were developed: a thruster with closed electron drift and extended acceleration zone, or Stationary Plasma Thruster (SPT), and a thruster with a very short acceleration channel, or Thruster with Anode Layer (TAL). The SPT employs a relatively long acceleration channel and ceramic wall insulator materials, such as boron nitride or silicon carbide. The TAL employs a shorter acceleration channel and conducting metallic wall materials which are typically stainless steel or molybdenum.

Among Hall thruster technologies, TAL, which was developed in the 1960's at TsNIIMASH, seems to be advantageous for two reasons. First, TAL has a very short acceleration zone (a few millimeters) and so there is less contact of ions with thruster surfaces; hence, it is favorable for long-term missions because of a reduction in erosion of thruster components. Second, higher power Hall thrusters will be needed in future space missions and a TAL with very high power has been specially developed to meet this requirement. ${ }^{1}$

Modeling of the plume fields yields important information in two different ways. First, it provides understanding of the plume impingement that involves fluxes of high-energy ions and charge-exchanged particles onto sensitive spacecraft devices such as solar arrays. Second, plume modeling helps to clarify the complex plasma processes inside the thruster with the aim of improving propulsion performance. The near field plume of a Hall thruster is a very important region because its high plasma density makes it relatively easy to use a variety of experimental diagnostic techniques. Such diagnostics are much more difficult to apply either in the internal thruster flow or in the plume far field. Therefore, understanding the behavior of the thruster plume is critical to the design of thrusters and spacecraft.

In the Hall thruster, the magnetic field in the acceleration channel provides the closed azimuthal electron drift and enhances the impact ionization of the propellant. Experimental measurements of the near-field plasma plume from Hall thrusters show that the magnetic field leaks into the plume and it is strong enough to affect the electron motion in the near-field plume region. ${ }^{2,3}$ However, among the rare reports of simulations for plume flows from a TAL, very few considered magnetic field effects. One of these reports performed quasi one dimensional simulations of the plasma plume from the D55 and indicated that the plasma potential increased with axial distance from the thruster exit plane. ${ }^{4}$ 
A plasma plume is a complex rarefied flow with several species: atoms, positively charged ions, and electrons. Generally, a hybrid particle-fluid approach is used for the computational simulation of plasma plume flow into vacuum. The direct simulation Monte Carlo (DSMC) method ${ }^{5}$ simulates the collisions of heavy particles (ions and atoms), and the Particle-In-Cell (PIC) technique ${ }^{6}$ models the transport of ions in electric fields. Electrons are treated using a fluid description, because electrons, which have significantly lighter mass, can adjust their velocities more quickly than ions or atoms.

For the electron fluid model, usually the Boltzmann relation is adopted. ${ }^{7,8}$ The Boltzmann model provides the plasma potential using several strong assumptions such as a constant electron temperature for a whole domain. The Detailed model which has been developed recently, ${ }^{9}$ is based on the conservation laws for electrons and is capable of representing accurate and detailed distributions for electron temperature, plasma potential and electron velocity. This model was successfully applied in a simulation of an axi-symmetric plasma plume from a 200W class SPT-type Hall thruster ${ }^{9}$ and in another simulation of 3D plasma plumes from a cluster of four $200 \mathrm{~W}$ class Hall thrusters. ${ }^{10}$

In this study, we analyze the possible influence of the magnetic field on the very near plasma plume. The 2D axisymmetric plume flow fields from a D55 TAL Hall thruster are investigated using MONACO, ${ }^{11}$ a hybrid PICDSMC code developed at the University of Michigan with both the Boltzmann model and the Detailed model. A stationary magnetic field is assumed.

The device considered in the present study is the D55 TAL Hall thruster developed by TsNIIMASH. We have chosen to study the D55 Hall thruster because of the availability of a significant amount of experimental data for this device.

Section II reviews numerical models, and brief information for experiments and flow conditions. Section III presents general features of the numerical 2D simulation results and a comparison of these results with experimental data taken in the plume of the D55.

\section{Models and Flow Conditions}

\section{A. Plasma Dynamics}

For particle simulation of plume flows, heavy neutrals and ions are modeled with the PIC-DSMC method, and the electrons are assumed as a fluid because electrons adjust their velocities more quickly with their significantly lighter mass. 
The simplest fluid electron model is the Botlzmann relation

$$
\phi=\phi_{r e f}+\frac{k T_{r e f}}{e} \ln \left(\frac{n_{e}}{n_{r e f}}\right)
$$

This equation is derived using several assumptions including that the electron flow is isothermal, collisionless, obeys the ideal gas law, and the magnetic field is neglected.

Recently, the Detailed model was proposed ${ }^{9}$ which represents a significantly increased level of physics compared to the Boltzmann model. In the Detailed model, the electron continuity equation is transformed into a Poisson equation by assuming steady flow and introducing a stream function; ${ }^{9}$

$$
\nabla^{2} \Psi=C_{i} e^{n_{a}}
$$

where $n_{e} \mathbf{V}_{\mathbf{e}}=\nabla \Psi$ and the xenon ionization rate coefficient $C_{i}$ is expressed as a function of electron temperature using a relation proposed by Ahedo ${ }^{12}$

$$
C_{i}=\sigma_{i} c_{e}\left(1+\frac{T_{e} \varepsilon_{i}}{\left(T_{e}+\varepsilon_{i}\right)^{2}}\right) \exp \left(-\frac{\varepsilon_{i}}{T_{e}}\right)
$$

In the presence of a magnetic field, assuming a steady state, neglecting the inertial term, the electron momentum equation is written ${ }^{13}$

$$
0=-e n_{e}\left(\mathbf{E}+\mathbf{V}_{e} \times \mathbf{B}\right)-\nabla p_{e}+n_{e} v_{e} m_{e}\left(\mathbf{V}_{\mathbf{e}}-\mathbf{V}_{\mathbf{i}}\right)
$$

Experimental investigation of the magnetic field distribution near a Hall thruster shows that the magnetic field has both radial and axial components of which the radial component is much larger. ${ }^{3}$ Thus, for simplicity, only the radial component of the magnetic field is considered. In this case, the plasma plume flow is across the magnetic field and the radial component of the magnetic field B varies along the axis. With another assumption that electrons behave as an ideal gas, Eq. (4) can be written in component form as:

$$
0=-e n_{e}\left(E_{z}-V_{e \phi} B_{r}\right)-k T \frac{d n_{e}}{d z}-n_{e} v_{e} m_{e}\left(V_{e z}-V_{i z}\right)
$$




$$
0=-e n_{e}\left(E_{z}-V_{e \phi} B_{r}\right)-k T \frac{d n_{e}}{d z}-n_{e} v_{e} m_{e}\left(V_{e z}-V_{i z}\right)
$$

From the azimuthal component,

$$
V_{e \phi}=-\frac{e}{m_{e} \nu_{e}} V_{e z} B_{r}+V_{i \phi} \approx-\frac{e}{m_{e} \nu_{e}} V_{e z} B_{r}
$$

However, $V_{e \phi}$ from Eq. (7) is derived when the density is high enough to make collisions occur. If the density is low then collisions are very rare, $V_{e \phi}$ becomes just a drift velocity, $V_{d r i f t}=E_{z} / B_{r}$.

So here we use

$$
V_{e \phi}=(1-w) V_{d r i f t}+w\left(-\frac{e}{m_{e} v_{e}} V_{e z} B_{r}\right)
$$

where weight function is $w=\left(\frac{n}{n_{\max }}\right)$ and $\mathrm{n}_{\max }$ is a local maximum which is chosen as a neutral particle density at the thruster exit.

A generalized Ohm's law is obtained from Eq. (4)

$$
\nabla \cdot \mathbf{j}=\nabla \cdot \sigma\left[-\nabla \phi+\mathbf{V}_{e} \times \mathbf{B}+\frac{1}{e n_{e}} \nabla\left(n_{e} k T_{e}\right)\right]
$$

where $-\nabla \phi=\mathbf{E}$.

From Eq. (9), an equation for the plasma potential can be obtained

$$
\begin{aligned}
& \nabla \cdot(\sigma \nabla \phi)=\frac{k \sigma}{e}\left(\nabla^{2} T_{e}+T_{e} \nabla^{2}\left(\ln n_{e}\right)\right)+\sigma \nabla\left(\ln n_{e}\right) \cdot \nabla T_{e}+T_{e} \nabla \sigma \cdot \nabla\left(\ln n_{e}+\nabla \sigma \cdot \nabla T_{e}\right) \\
& -\frac{\partial \sigma}{\partial z} V_{\phi} B_{r}-\sigma\left[V_{\phi} \frac{\partial B_{r}}{\partial z}+B_{r} \frac{\partial V_{\phi}}{\partial z}\right]
\end{aligned}
$$

For given $n_{e}, v_{e}, T_{e,}$ and $V_{\phi}$ the charge continuity condition

$$
\nabla \cdot \mathbf{j}=0
$$

is solved to obtain the plasma potential. 
The electron temperature equation is obtained from the steady state electron energy equation ${ }^{14}$

$$
\nabla^{2} T_{e}=-\nabla \ln \kappa_{e} \cdot \nabla T_{e}+\frac{1}{\kappa_{e}}\left(-\mathbf{j} \cdot \mathbf{E}+\frac{3}{2} n_{e}\left(\mathbf{V}_{\mathbf{e}} \cdot \nabla\right) k T_{e}+p_{e} \nabla \cdot \mathbf{V}_{\mathbf{e}}+3 \frac{m_{e}}{m_{i}} v_{e} n_{e} k\left(T_{e}-T_{H}\right)+n_{e} n_{a} C_{i} \varepsilon_{i}\right)
$$

Finally, the electron conductivity $\sigma$ and the electron thermal conductivity $\kappa_{e}$ can be evaluated using their basic definitions $^{14}$

$$
\begin{gathered}
\sigma=\frac{e^{2} n_{e}}{m_{e} v_{e}} \\
\kappa_{e}=\frac{2.4}{1+\frac{v_{e i}}{\sqrt{2} v_{e}}} \frac{k^{2} n_{e} T_{e}}{m_{e} v_{e}}
\end{gathered}
$$

where $v_{e}=v_{e i}+v_{e n}, v_{e i}$ is the ion-electron collision frequency, $v_{e n}$ is the neutron-electron collision frequency and these frequencies are evaluated for the xenon system using cross sections provided in ref. 14 .

By treating the right hand side terms as known sources and solving Eqs. (2), (11) and (12) three fundamental fluid electron properties are obtained, i.e., electron velocity, plasma potential, and electron temperature.

\section{B. Collision Dynamics}

Two types of collisions are important in the Hall thruster: elastic (momentum exchange: MEX) and charge exchange (CEX). There are two kinds of elastic collisions: atom-atom and atom-ion interactions. For atom-atom collisions, the Variable Hard Sphere ${ }^{5}$ model is used and the collision cross section for xenon is

$$
\sigma_{e l}(\mathbf{X e}, \mathbf{X e})=\frac{2.12 \times 10^{-18}}{g^{2 \omega}}
$$

where $\mathrm{g}$ is the relative velocity and $\omega=0.12$ is related to the viscosity temperature exponent for xenon. For atomion elastic collisions, the MEX cross section is set equal to the CEX cross section.

Charge exchange concerns the transfer of one or more electrons between an atom and an ion. For single charged ions, we use the following cross section measured by Pullins et al. ${ }^{15}$ and Miller et al. ${ }^{16}$

$$
\sigma_{c e x}\left(\mathbf{X e}, \mathbf{X e}^{+}\right)=1.1872 \times 10^{-20}(142.21-23.30 \log (g))
$$


Also, Refs. 15 and 16 reported that the CEX cross section for double charged ions is approximately half as large as single charged ions at corresponding energies.

\section{Flow Conditions}

A schematic of the D55 thruster is presented in Fig. 1. The D55 thruster has an annular anode chamber with a mean diameter of $55 \mathrm{~mm}$ and a width of $5 \mathrm{~mm}$.

Figure 2 shows contours of the radial component of the magnetic field outside of the thruster which are reconstructed using experimental data. ${ }^{2}$ We extrapolated data from $0 \mathrm{~mm}$ to $5 \mathrm{~mm}$ where experimental data are not available. Because of the proprietary nature of the magnetic field data, the values reported in this paper have been normalized to the maximum value.

We consider two different experimental conditions. Most of the results presented here are for a series of experiments conducted at the University of Michigan. ${ }^{2}$ The D55 thruster was operated at a flow rate of $4.76 \mathrm{mg} / \mathrm{s} \mathrm{of}$ xenon, a discharge voltage of $300 \mathrm{~V}$, and a current of $4.5 \mathrm{~A}$. The specific impulse under these conditions was previously measured to be $1,810 \mathrm{~s} .^{17}$ For the D55 thruster, it is known that a portion of the thrust is generated outside the thruster, so we assumed that thrust from the thruster itself is $80 \%$ of the total thrust in order to estimate the appropriate thruster exit velocity. The number fraction of double xenon ions is assumed to be 0.25 . At the thruster exit, the electron temperature is taken to be $10 \mathrm{eV}$ in the Boltzmann model and the Detailed model to obtain good agreement between the simulations and the data measured in the plume field, the temperature of the ions is assumed to be $4 \mathrm{eV}$, and that of the neutrals is assumed to be $750 \mathrm{~K}$. The backpressure in the Michigan facility is reported as $8.3 \times 10^{-3} \mathrm{~Pa}$.

The second flow condition corresponds to a study performed by the University of Tennessee Space Institute (UTSI) and Lockheed Martin Astronautics (LMA). ${ }^{18}$ The thruster was operated at a flow rate of $6 \mathrm{mg} / \mathrm{s}$ and a current of 4.5 A. The background pressure with the thruster running was $9.3 \times 10^{-3} \mathrm{~Pa}$.

The D55 has a nozzle-like geometry at the exit, so the plume spreads at the thruster exit with certain angles. In the present study, we adopt $15 \mathrm{deg}$ as a half angle, and the radial velocity varies linearly across each half of the exit plane. $^{8}$

The computational grid employed in the present study consists of rectangular cells. The smallest cells are located close to the thruster exit and have a size of $2.5 \mathrm{~mm}$. The largest cells are those close to the edges of the domain and have a size of $5 \mathrm{~mm}$. The domain size is $0.2 \mathrm{~m}$ by $0.26 \mathrm{~m}$. The computations presented in the study typically 
employed 300,000 particles with a total of 60,000 time steps. Table 1 is a listing of the flow conditions assumed at the thruster exit.

\section{Results}

Overall plasma potential fields obtained with the Boltzmann model and the Detailed model are presented in Fig 3. For both models, we set $145 \mathrm{~V}$ as the plasma potential at the channel exit to obtain good agreement between measured and simulated data, especially for radial profiles of ion current density and axial components of velocity in the very near field plume. Fig. 3 shows the Boltzmann model results, and Fig. $4 \mathrm{a}$ and $4 \mathrm{~b}$ show the Detailed model results without and with the magnetic field, respectively. It is reported that the plasma potential gradient of the Boltzmann model and the Detailed model are very different. From Fig. 3 and 4a we can see this feature. From Fig 4a and $4 \mathrm{~b}$, one can see that if the magnetic field is considered that the plasma potential gradients become stronger. It is expected that only electrons are magnetized while ion motion does not feel any significantly effects from the magnetic field. However, plasma flow across the magnetic field under this condition generates additional electric fields which act to increase the total fields. One possible reason is that the confinement of the electron motion by the magnetic field reduces the electric conductivity which results in increasing the electric field.

A series of probe experiments was performed by Domonkos et al. ${ }^{2}$ in the near field of the D55 plume. The local plasma potential was obtained using an emissive probe and a Langmuir probe; ion current density was obtained using a Faraday probe; and the electron temperature and number density were obtained using a Langmuir probe.

Figures $5 \mathrm{a}$ and $5 \mathrm{~b}$ show radial profiles of plasma potential at axial distances of $10 \mathrm{~mm}$ and $50 \mathrm{~mm}$ from the thruster exit plane, respectively. Experimental data were measured with respect to the cathode potential of $14 \mathrm{~V},{ }^{2}$ so here we added $14 \mathrm{~V}$ to the measured data for consistency with the simulation. Close to the thruster, the Boltzmann model overpredicts the potential. The Detailed model captures the shape quite well although it overpredicts the potential too. However the result is better than the Boltzmann model and if the magnetic field is considered the improvement is remarkable. At $50 \mathrm{~mm}$ from the thruster, the Boltzmann model still greatly overpredicts the potential, but the Detailed model without the magnetic field results and measured data are in better agreement and the magnetic field consideration case gives best results.

Ion current density profiles predicted by the simulation are compared with the experimental data ${ }^{2}$ in Figs. $6 \mathrm{a}$ and $6 \mathrm{~b}$ along radial lines located 10 and $40 \mathrm{~mm}$ from the thruster exit plane, respectively. Both the Boltzmann model and 
the Detailed model give good prediction at $10 \mathrm{~mm} .40 \mathrm{~mm}$, the two models slightly underpredict the results but are still in good agreement. This underprediction of the current density implies a possibility that the simulation overaccelerates ionized particles in the radial direction between 10 and $40 \mathrm{~mm}$ from the thruster exit plane. The variation of the ion current density with axial distance from the thruster indicates that the ion flow begins as an annulus and then merges to a cylinder-shaped beam. This feature is because the annular ion flow is diverging with an angle of 15 degrees so overlaps at the centerline of the thruster as the flow goes downstream. One can see that the magnetic field consideration does not give much change in the ion current density.

Measurements of electron number density ${ }^{2}$ are compared with the simulations for radial profiles at 10 and 50 $\mathrm{mm}$ in Figs. $7 \mathrm{a}$ and $7 \mathrm{~b}$, respectively. The simulation values represent the total charge density obtained from the number densities of the $\mathrm{Xe}^{+}$and $\mathrm{Xe}^{++}$ions. The measured data have an accuracy of \pm 50 percent at 10 and $50 \mathrm{~mm}$. Therefore, in the near field, most of the simulation data are underpredict the measured values. The peak electron number density measured at both stations is more than double the total charge density assumed in the simulations at the thruster exit plane (see Table 1). Gulczinski et al. ${ }^{19}$ presented the evidence that the Langmuir probe technique leads to high electron number densities. At a distance of $25 \mathrm{~cm}$ and $50 \mathrm{~cm}$ from the thruster, the Langmuir probe gave six times higher electron number density than the microwave interferometer. ${ }^{19}$ One other possible interpretation of these comparisons is that the axial component of electric fields in the simulation is so strong that the acceleration of ions is overestimated in the axial direction. One possible way to address such differences between the model and the measured data would be axial confinement of electrons caused by the magnetic field. It was shown that the axial component of the magnetic field is much smaller than the radial component of that in SPT100 thrusters, ${ }^{4}$ there is no direct evidence that it is true in D55 thrusters. Measurements of axial component of the magnetic field are required to help resolve this issue, and no such data exist as of now.

Figures $8 \mathrm{a}$ and $8 \mathrm{~b}$ show radial profiles of electron temperature at distances of 10 and $50 \mathrm{~mm}$ from the thruster, respectively. The experimental uncertainty is reported to be $\pm 10 \%{ }^{2}$ At $10 \mathrm{~mm}$, it is clear from the measurement that there is significant variation in the electron temperature because of the dynamics of the plasma inside of the acceleration channel. Electron temperature is constant in the Boltzmann model and here we show the value of $10 \mathrm{eV}$ which is used in the simulation. In general, although the Detailed model provides reasonable agreement with the measurements, the radial gradients predicted by the model are smaller than the measured data indicate. These disparities between the models and the measurement indicate that more elaborated thruster exit boundary conditions 
are needed. In fact, it is know that the Detailed model is relatively more sensitive to boundary conditions than the Boltzmann model.

Finally, the simulation results are compared with Laser Induced Fluorescence (LIF) measurements of $\mathrm{Xe}^{+}$axial velocity component obtained by Keefer et al. ${ }^{18}$ in Fig. 8. In Ref. 18, it is explained that the reported velocity data represent the central value of the ion velocity distribution functions detected by the LIF diagnostic. Therefore, for consistency with the experiment, the ion velocity distribution function is calculated throughout the flow field, and the most probable value of the distributions obtained. Figure 9 shows the axial velocity profiles at a radial position of $27.5 \mathrm{~mm}$ which is along the thruster channel center. The simulations use the second set of operating conditions given in Table 1. It is clear that the Boltzmann model fails to produce sufficient ion acceleration in the near field of the plume. This is an expected result because the plasma potential gradient is not quite enough to give ions significant acceleration in Fig. 3. As discussed with reference to Fig. 4, the Detailed model predicts strong ion acceleration in the near field region and rapidly accelerates the ions from the thruster exit velocity of $15 \mathrm{~km} / \mathrm{s}$ to a value of about $18 \mathrm{~km} / \mathrm{s}$ that corresponds to the measured data and the results becomes slightly better when the magnetic field is considered. The simulation result, however, overstimates the axial velocity at $\mathrm{z}=1 \mathrm{~mm}$. This discrepancy also suggests that improved thruster exit flow conditions are needed.

\section{Conclusion}

A hybrid particle-fluid PIC-DSMC model using both a Detailed model and the Boltzmann model for the fluid electrons was applied to simulate the plume flow from a D55 anode layer Hall thruster. The present model considers the plasma plume region where the magnetic field leaked from the acceleration channel may have a substantial effect on the plasma potential distribution. Analyses of the plasma potential distribution across the magnetic field show that the field significantly affects the profile of the plasma in the Detailed model. For instance, in the case of zero magnetic field, the plasma has a potential of about $110 \mathrm{~V}$ at $10 \mathrm{~mm}$ from the thruster exit, while in the case of a finite magnetic field, the plasma potential is about $80 \mathrm{~V}$. Results predicted by the Detailed model with the magnetic field are found to be in better agreement with experimental data. Generally, the Detailed model provided better results than the Boltzmann model. The inclusion of the magnetic field gives closer prediction of the plasma potential distribution to the measured one in the Detailed model. The Detailed model with the magnetic field consideration more accurately predicted the extended ion acceleration region outside the thruster. By comparison, the Boltzmann 
model indicated almost no ion acceleration outside the thruster. The simulation results of the Detailed model and the Boltzmann model underpredicted the electron number density. This disparity may be caused by the simulated electric fields being too strong and leading to overacceleration of the ions in the axial direction. This work will be extended to the far-field plume and comparison with measurement. Future work will also involve modeling the D55 thruster plasma to generate improved thruster exit flow conditions.

\section{Acknowledgments}

This work is supported by AFOSR Grant FA9550-05-1-0042 with Dr. Mitat Birkan as technical monitor. The authors gratefully acknowledge the contributions to this work by Dr. Chunpei Cai for discussions.

\section{References}

${ }^{1}$ Marrese, C. M., Frisbee, R., Sengupta, A., Cappelli, M. A., Tverdoklebov, S., Semenkin, S., and Boyd, I. D., "Very High $\mathrm{I}_{\mathrm{sp}}$ Thruster with Anode Layer (VHITAL): An Overview,” AIAA Paper 2004-5910, July 2004.

${ }^{2}$ Domonkos, M. T., Marrese, C. M., Haas, J. M., and Gallimore, A. D., "Very Near-Field Plume Investigations of the D55," AIAA Paper 97-3062, July 1997.

${ }^{3}$ Oh, D. Y., Hastings, D. E., Marrese, C. M., Haas, J. M., Gallimore, A. D., "Modeling of Stationary Plasma Thruster-100 Thruster Plumes and Implications for Satellite Design”, Journal of Propulsion and Power, Vol. 15, No 2, 1999, pp. 345-357.

${ }^{4}$ Keidar, Michael, Boyd, I. D., "Effect of a Magnetic Field on the Plasma Plume from Hall Thrusters", Journal of Applied Physics, Vol. 86, 1999, pp. 4786-4791.

${ }^{5}$ Bird, G. A., Molecular Gas Dynamics and the Direct Simulation of Gas Flows, Oxford Press, New York, 1994.

${ }^{6}$ Birdsall, C. K., and Langdon, A. B., Plasma Physics Via Computer Simulation, Adam Hilger, New York, 1991.

${ }^{7}$ Keidar, M., Choi, Y., and Boyd, I. D., "Modeling a Two-Stage High-Power Bismuth Anode Layer Thruster and its Plume," IEPC Paper 2005-045, October 2005.

${ }^{8}$ Boyd, I. D., "Computation of the Plume of an Anode-Layer Hall Thruster," Journal of Propulsion and Power, Vol. 16, No. 5, 2000, pp. 902-909.

${ }^{9}$ Boyd, I. D. and Yim, J. T., "Modeling of the Near Field Plume of a Hall Thruster," Journal of Applied Physics, Vol. 95, 2004, pp. 4575-4584. 
Cai, C., and Boyd, I. D., “3D Simulation of Plume Flows from a Cluster of Plasma Thrusters," AIAA Paper 2005-4662, July 2005.

11 Dietrich, S. and Boyd, I. D., "Scalar and Parallel Optimized Implementation of the Direct Simulation Monte Carlo Method", J. of Computational Physics, Vol. 126, 1996, pp. 328-342.

12 Ahedo, E., Martinez-Cerezo, P., and Martinez-Sanchez, M., "One-dimensional Model of the Plasma Flow in a Hall Thruster," Physics of Plasma, Vol. 8, 2001, pp. 3058-3068.

13 Braginsky, S. I., in Reviews of the Plasma Physics edited by Leontovich, M. A., (Consultants Bureau, New York, 1965), Vol. 1.

14 Mitcher, M., and Kruger, C. H., Partially Ionized Gases, Wiley, 1973.

15 Pullins, S. H., Chiu, Y., Levandier, D. J., and Dresseler, R. A., "Ion Dynamics in Hall Effect and Ion Thruster Xenon Symmetric Charge Transfer,” AIAA Paper 2000-0636, January 2000.

16 Miller, S., Levandier, D. J., Chiu, Y., and Dresseler, R. A., "Xenon Charge Exchange Cross Sections for Electrostatic Thruster Models," Journal of Applied Physics, Vol. 19, 2002, pp. 984-991.

17 Semenkin, A., Kochergin, A., Garkusha,V., Chislov, G., Rusakov, A., Tverdoklebov, S., and Sota, C., “RHETT/EPDM Flight Anode Layer Thruster Development,” IEPC Paper 97-106, August 1997

18 Keefer, D., Wright, N., Hornkohl, J. O., and Bangasser, J., "Multiplexed LIF and Langmuir Probe Diagnostic Measurements in the TAL D-55 Thruster," AIAA Paper 97-2425, July 1999.

19 Gulczinski, F. S., Gallimore, A. D., Carlson, D. O., and Gilchrist, B. E., "Impact of Anode Layer Thruster Plumes on Satellite Communications," AIAA Paper 97-3067, July 1997. 
Table 1 Flow properties assumed at thruster exit plane

\begin{tabular}{lllrr}
\hline \hline Experiment & species & $\mathrm{n} \mathrm{m}^{-3}$ & $\mathrm{~T}, \mathrm{~K}$ & $\mathrm{U}, \mathrm{m} / \mathrm{s}$ \\
\hline Michigan & $\mathrm{Xe}$ & $1.1 \times 10^{18}$ & 750 & 281 \\
& $\mathrm{Xe}^{+}$ & $4.0 \times 10^{17}$ & 46,400 & 15,000 \\
& $\mathrm{Xe}^{++}$ & $1.0 \times 10^{17}$ & 46,400 & 21,300 \\
UTSI \& LMA & $\mathrm{Xe}$ & $8.9 \times 10^{18}$ & 750 & 281 \\
& $\mathrm{Xe}^{+}$ & $4.0 \times 10^{17}$ & 46,400 & 15,000 \\
& $\mathrm{Xe}^{++}$ & $1.0 \times 10^{17}$ & 46,400 & 21,300 \\
\hline \hline
\end{tabular}

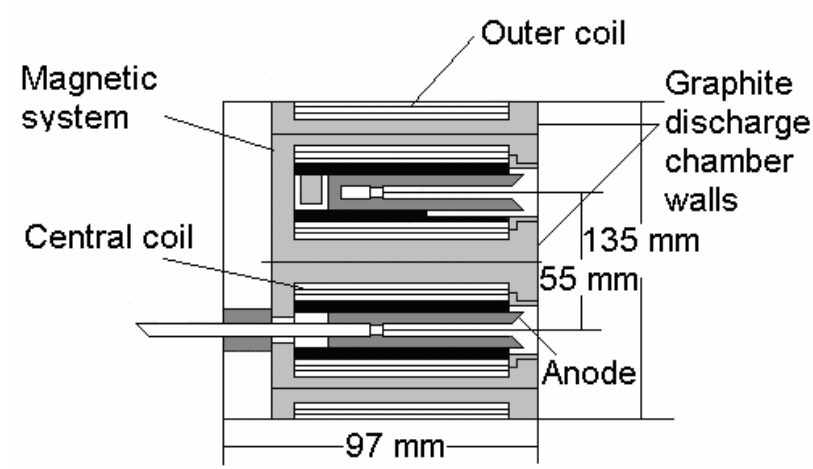

Figure 1. Schematic of the D55 anode-layer Hall thruster.

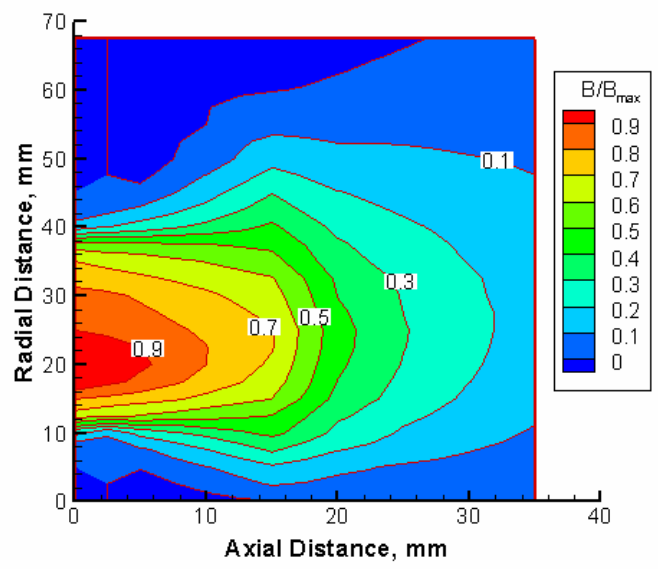

Figure 2. Magnetic field Profiles. 


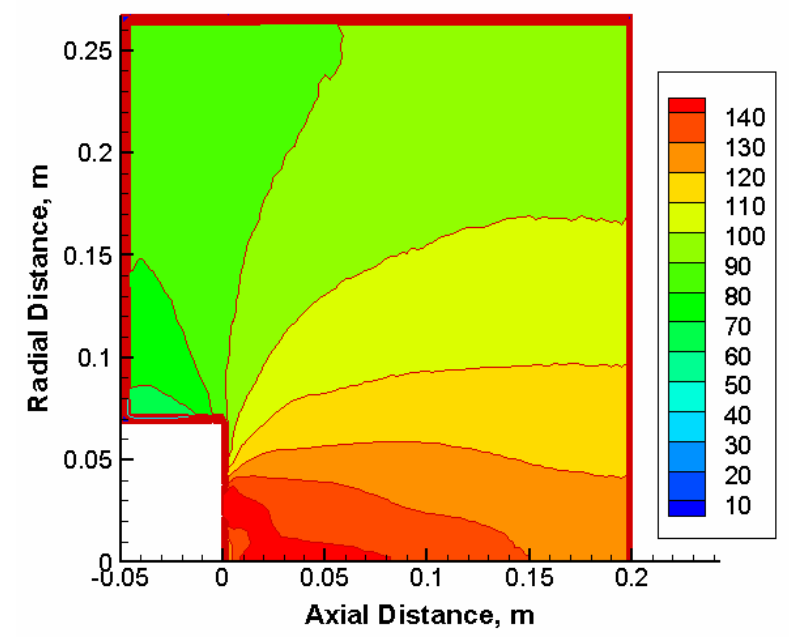

Figure 3. Contours of Plasma potential (in V), the Botlzmann model

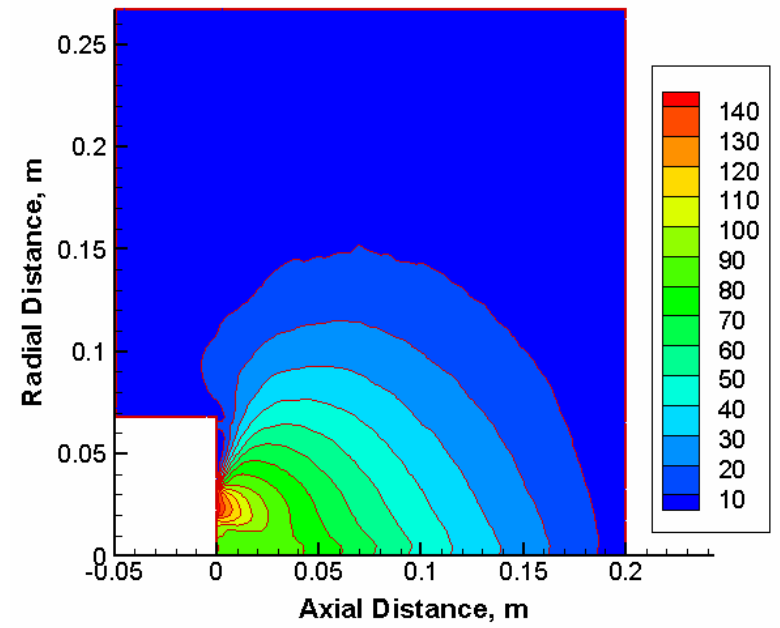

(a) without magnetic field

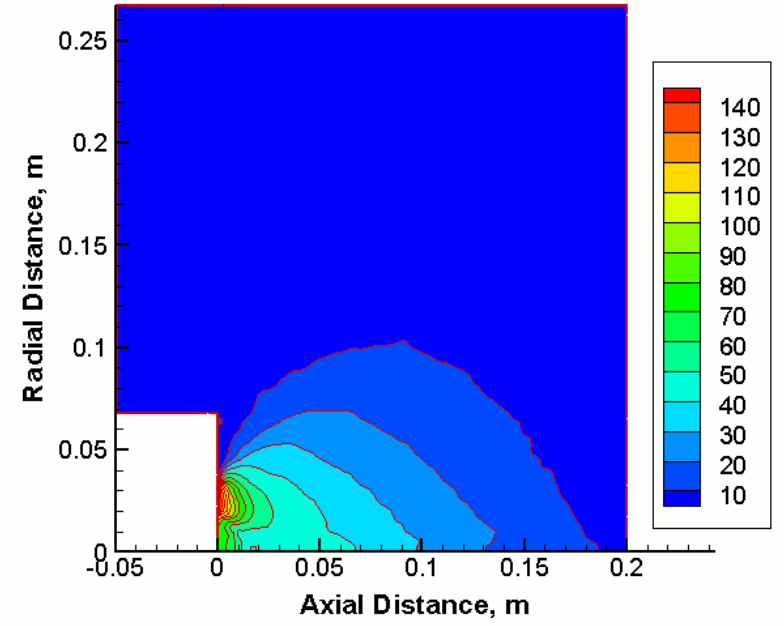

(b) with magnetic field

Figure 4. Contours of Plasma potential (in V), the Detailed model 


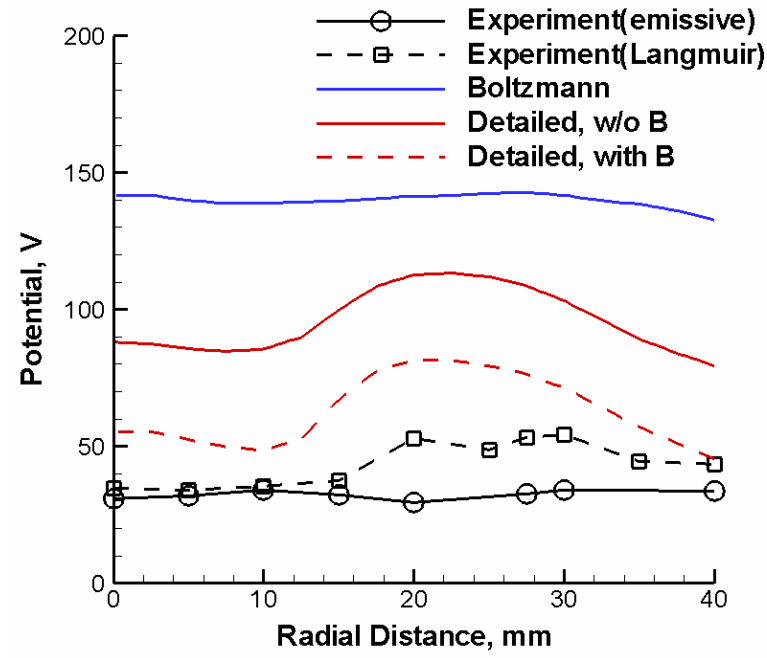

(a) $10 \mathrm{~mm}$ from the thruster exit plane.

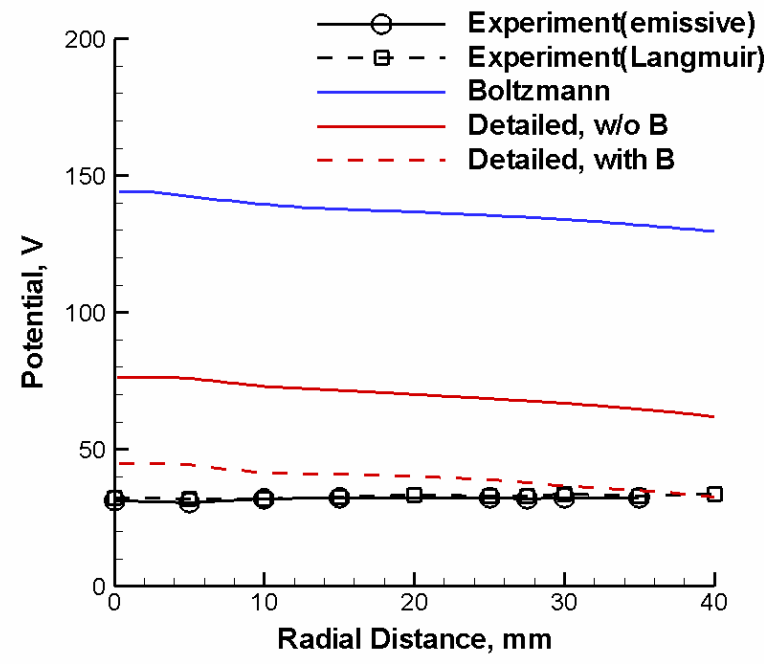

(b) $50 \mathrm{~mm}$ from the thruster exit plane.

Figure 5. Radial profiles of plasma potential.

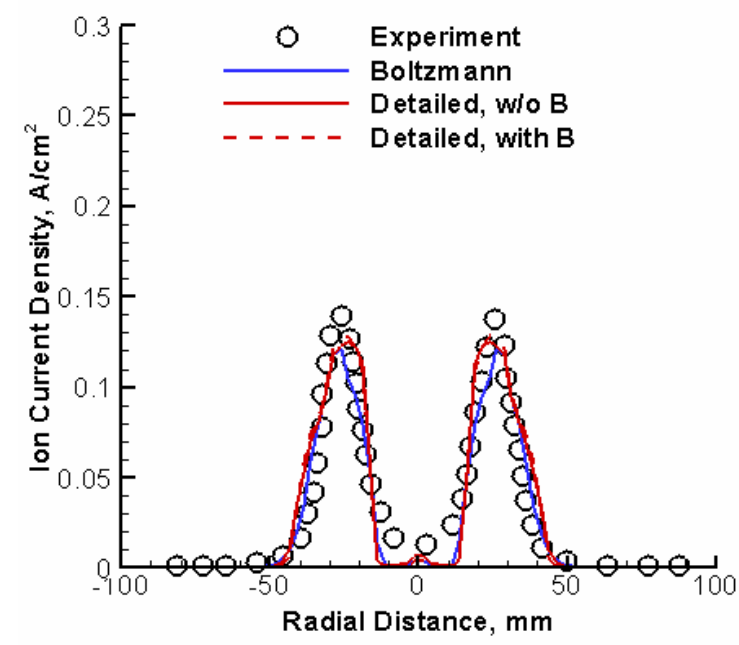

(a) $10 \mathrm{~mm}$ from the thruster exit plane.

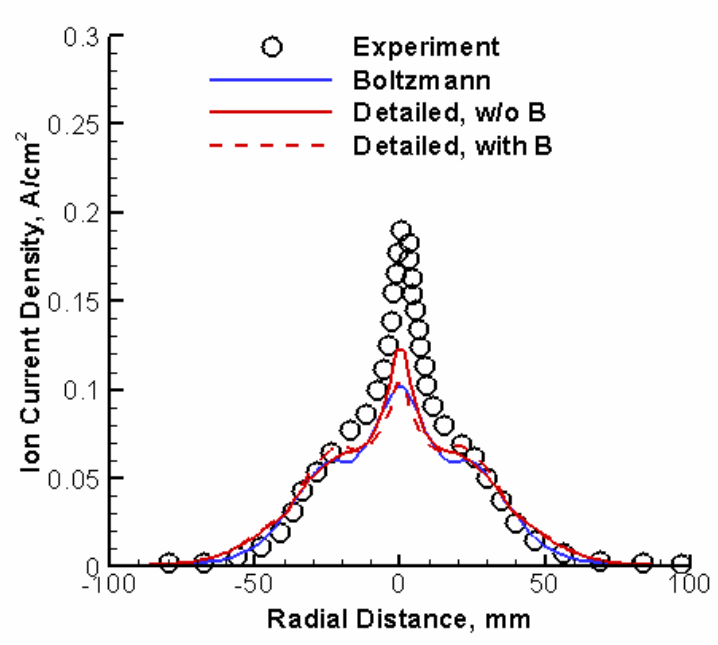

(b) $40 \mathrm{~mm}$ from the thruster exit plane.

Figure 6. Radial profiles of ion current density 

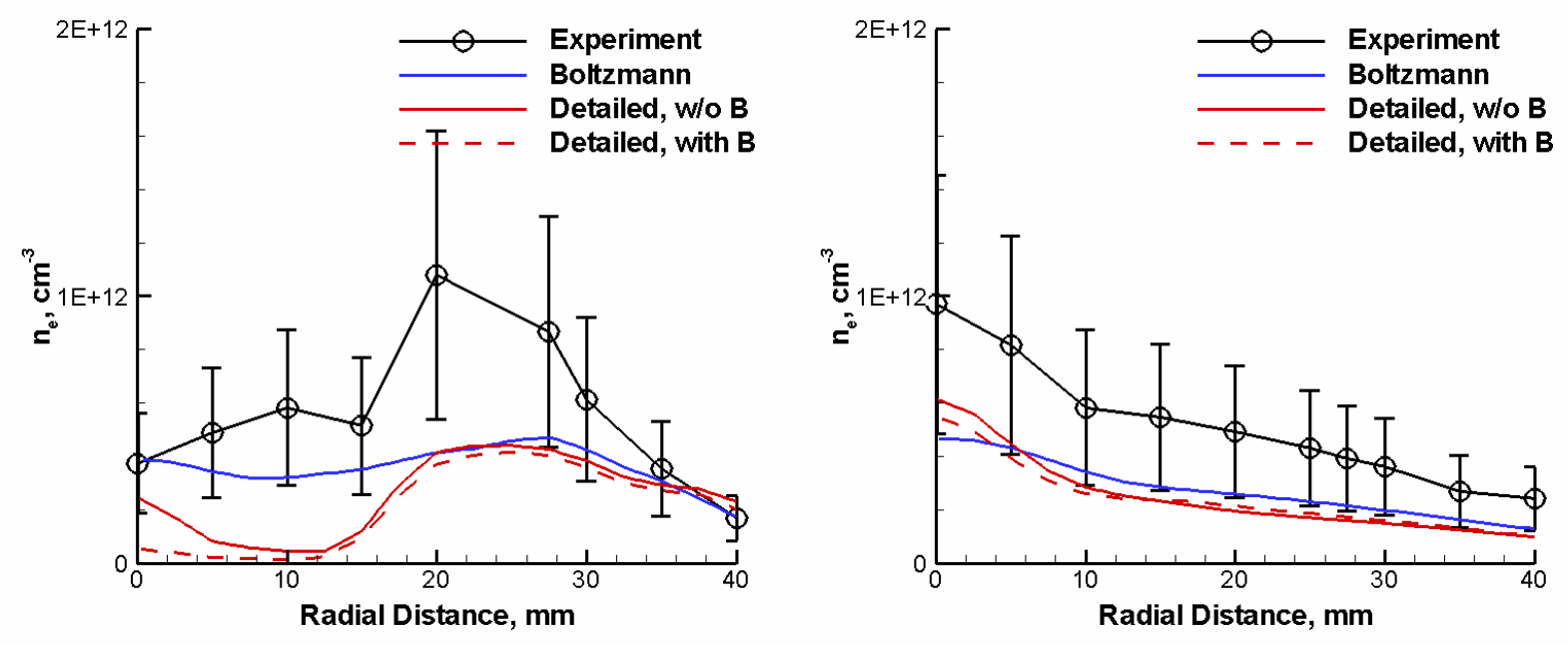

(a) $10 \mathrm{~mm}$ from the thruster exit plane.

(b) $40 \mathrm{~mm}$ from the thruster exit plane.

Figure 7. Radial profiles of electron number density

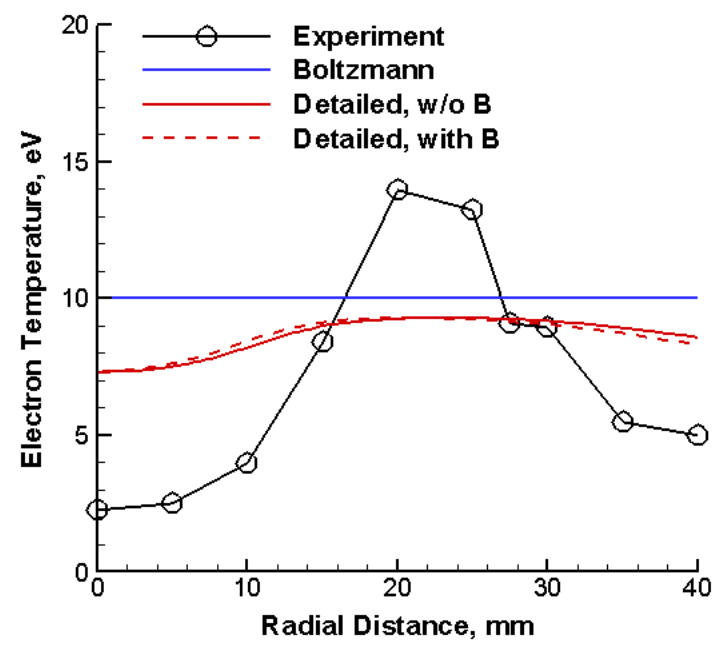

(a) $10 \mathrm{~mm}$ from the thruster exit plane.

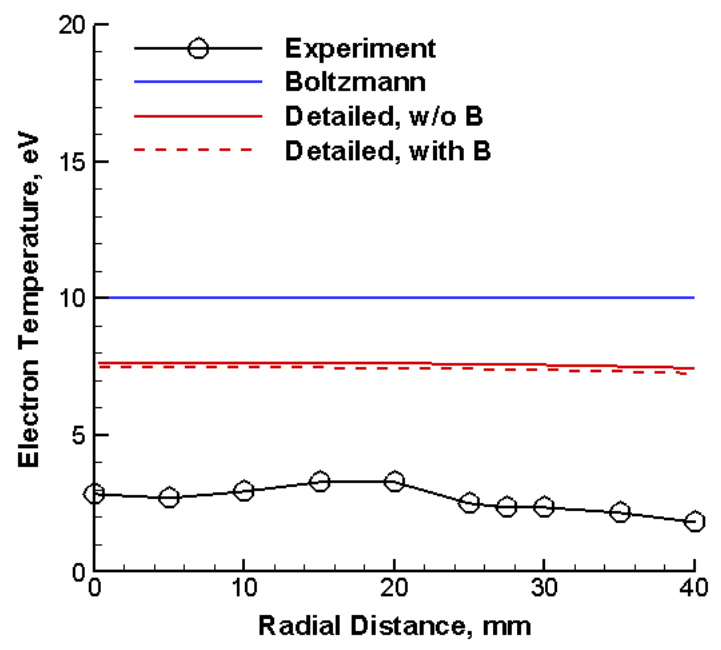

(b) $50 \mathrm{~mm}$ from the thruster exit plane.

Figure 8. Radial profiles of electron temperature 


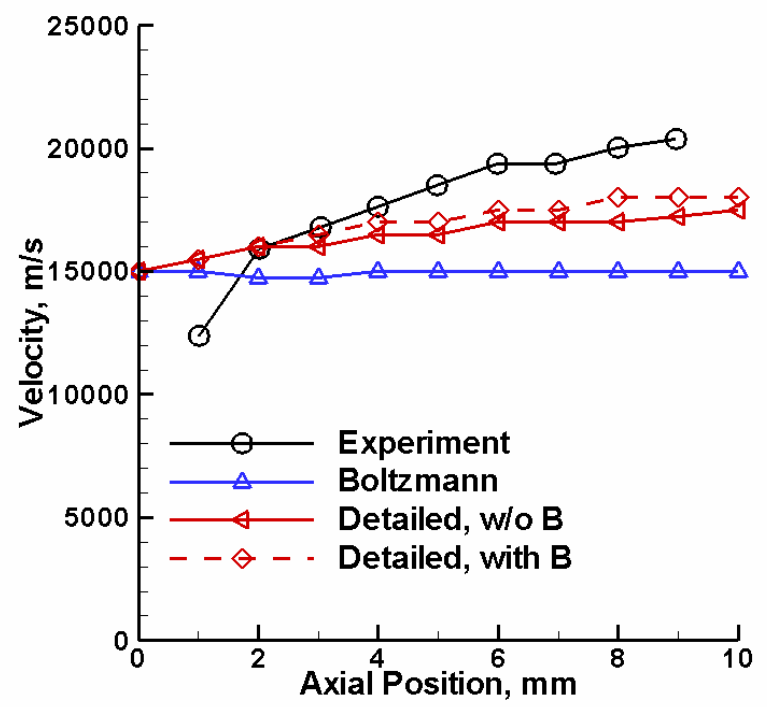

Figure 9. Axial components of velocity at a radial position of $27.5 \mathrm{~mm}$. 